\title{
Impact of Plasmodium falciparum gene deletions on malaria rapid diagnostic test performance
}

\author{
Michelle L. Gatton ${ }^{*} \mathbb{D}$, Alisha Chaudhry', Jeff Glenn², Scott Wilson², Yong Ah², Amy Kong ${ }^{3}$, Rosalynn L. Ord ${ }^{4}$, \\ Roxanne R. Rees-Channer ${ }^{4}$, Peter Chiodini ${ }^{4,5}$, Sandra Incardona ${ }^{6}$, Qin Cheng ${ }^{7}$, Michael Aidoo ${ }^{3}$ \\ and Jane Cunningham ${ }^{8}$
}

\begin{abstract}
Background: Malaria rapid diagnostic tests (RDTs) have greatly improved access to diagnosis in endemic countries. Most RDTs detect Plasmodium falciparum histidine-rich protein 2 (HRP2), but their sensitivity is seriously threatened by the emergence of pfhrp2-deleted parasites. RDTs detecting P. falciparum or pan-lactate dehydrogenase (Pf- or pan$\mathrm{LDH}$ ) provide alternatives. The objective of this study was to systematically assess the performance of malaria RDTs against well-characterized pfhrp2-deleted P. falciparum parasites.

Methods: Thirty-two RDTs were tested against 100 wild-type clinical isolates (200 parasites/ $\mu \mathrm{L}$ ), and 40 samples from 10 culture-adapted and clinical isolates of pfhrp2-deleted parasites. Wild-type and pfhrp2-deleted parasites had comparable Pf-LDH concentrations. Pf-LDH-detecting RDTs were also tested against 18 clinical isolates at higher density (2,000 parasites/ $\mu \mathrm{L}$ ) lacking both pfhrp2 and pfhrp3.

Results: RDT positivity against pfhrp2-deleted parasites was highest (>94\%) for the two pan-LDH-only RDTs. The positivity rate for the nine Pf-LDH-detecting RDTs varied widely, with similar median positivity between doubledeleted (pfhrp2/3 negative; 63.9\%) and single-deleted (pfhrp2-negative/pfhrp3-positive; 59.1\%) parasites, both lower than against wild-type P. falciparum (93.8\%). Median positivity for HRP2-detecting RDTs against 22 single-deleted parasites was 69.9 and 35.2\% for HRP2-only and HRP2-combination RDTs, respectively, compared to 96.0 and $92.5 \%$ for wild-type parasites. Eight of nine Pf-LDH RDTs detected all clinical, double-deleted samples at 2,000 parasites/uL.

Conclusions: The pan-LDH-only RDTs evaluated performed well. Performance of Pf-LDH-detecting RDTs against wild-type P. falciparum does not necessarily predict performance against pfhrp2-deleted parasites. Furthermore, many, but not all HRP2-based RDTs, detect pfhrp2-negative/pfhrp3-positive samples, with implications for the HRP2-based RDT screening approach for detection and surveillance of HRP2-negative parasites.
\end{abstract}

Keywords: Rapid diagnostic tests, Hisidine rich protein 2, HRP2, Gene deletion

\section{Background}

Antigen-detecting rapid diagnostic tests (RDTs) are recommended diagnostic tools by the World Health Organization (WHO) for malaria case management [1]. The

\footnotetext{
*Correspondence: m.gatton@qut.edu.au

${ }^{1}$ Queensland University of Technology, Brisbane, QLD, Australia

Full list of author information is available at the end of the article
}

implementation of malaria RDTs has greatly improved access to diagnosis in endemic countries, particularly in Africa [2].

In general, three types of RDTs for detection of Plasmodium falciparum are commercially available: 1) $P$. falciparum-only RDTs; 2) combination RDTs, which detect and differentiate $P$. falciparum and some, or all, non-P. falciparum species; and, 3) Pan-only RDTs, which 
detect but do not differentiate between $P$. falciparum and non- $P$. falciparum species. Most $P$. falciparum-detecting RDTs use histidine-rich protein 2 (HRP2) as it is speciesspecific and abdundantly produced. Some HRP2-based RDTs may also potentially detect $P$. falciparum histidine-rich protein 3 (HRP3) due to its structural similarity with HRP2 [3]. RDT bands detecting non-falciparum Plasmodium target Pan or species-specific lactate dehydrogenase (Pan-LDH, Plasmodium vivax (Pv)-LDH or $P$. vivax, Plasmodium ovale, Plasmodium malariae (Pvom)LDH), or aldolase. P. falciparum-LDH (Pf-LDH) can also be used specifically to detect $P$. falciparum. HRP2-based RDTs generally exhibit superior performance, particularly at low parasite densities, and are more heat stable than non-HRP2-based RDTs [4].

The sensitivity of HRP2-based RDTs is seriously threatened by the increasing occurrence of $P$. falciparum with deleted HRP2 and/or HRP3 antigen-coding genes. Plasmodium falciparum isolates lacking $p$ fhrp $2 / 3$ were first reported in Peru with a prevalence of 20 to $90 \%$, depending on location [5-7]. Subsequently, pfhrp2/3-deleted parasites have been reported in Colombia, Suriname, Bolivia, Brazil, Honduras, Guatemala, and Nicaragua [8-12]. Parasites lacking $p f h r p 2$ have also been reported around the China-Myanmar border [13] and in India [14] with prevalence up to $25 \%$ in some areas [15].

In Africa, parasites lacking one or both pfhrp 2 and pfhrp3 have been reported in Mali [16], Ghana [17], Senegal [18], Democratic Republic of the Congo [19], Rwanda [20], Zambia [21], and Kenya [22] with prevalences ranging between 2 and $45 \%$, while up to $80 \%$ of symptomatic patients at two regional hospitals in Eritrea had P. falciparum lacking pfhrp $2 / 3$ [23].

The emergence of parasites that do not express HRP2 poses a major public health threat due to the heavy reliance of RDTs on this antigen. In response, WHO has released a Response Plan [24]. If HRP2-based P. falciparum-only RDTs are used when a patient is infected solely with parasites lacking HRP2 then a false-negative diagnosis can occur, delaying correct treatment and potentially leading to severe complications and death. In regions where HRP2-pan-LDH combination tests are used, incorrect diagnosis of non-falciparum malaria can occur when individuals are infected with HRP2-negative P. falciparum, potentially impacting the treatment regimen and patient health outcome. In both situations, routine surveillance estimates of malaria incidence will be adversely affected.

The current solution to the diagnostic problem posed by $P$. falciparum parasites lacking $p f h r p 2 / 3$ is first to establish prevalence and, based on these results, decide if a replacement RDT or microsopy is needed. Any replacement RDT should not exclusively rely on HRP2 for $P$. falciparum. However, it can be challenging to maintain access to quality-assured microscopy and to switch RDTs, especially as the number of RDTs targeting alternative antigens is limited. Eighty-nine RDTs that detect $P$. falciparum, alone or in combination, have undergone WHO product testing within the past 5 years [4]. Of these, 78 use HRP2 exclusively to detect $P$. falciparum, with nine currently pre-qualified by WHO. The remaining $11 \mathrm{RDTs}$ use Pf-LDH either alone or in combination with HRP2, or pan-LDH only, to detect $P$. falciparum, with only two of these products (one pan-LDH and one HRP2/Pf-LDH) being WHO pre-qualified [4]. During WHO product testing, the performance of Pf-LDH test bands has been generally poor with only two products having met the WHO performance criteria based on Pf-LDH test line results against wild-type, HRP2-expressing $P$. falciparum.

Although many studies have evaluated RDT performance in the field, and the WHO and Foundation for Innovative New Diagnostics, in collaboration with the US Centers for Disease Control and Prevention (CDC), have led rigorous performance testing of RDTs over the past decade, there has been no systematic assessment of the performance of RDTs against well-characterized pfhrp2deleted $P$. falciparum parasites.

To address this gap, Round 8 WHO Malaria RDT Product Testing Programme included an evaluation of RDTs against a panel of $p$ fhrp2-deleted P. falciparum parasites, with and without $p f h r p 3$. Here, the findings are described and discussed with reference to the implications for future RDT use.

\section{Methods \\ Parasite samples}

Two panels of $P$. falciparum were tested against all RDTs in this study: 1) wild-type panel of 100 clinical pfhrp2positive isolates, and 2) pfhrp2-deleted panel containing 40 samples from 10 different isolates/strains (Table 1). All samples were genotyped for $p f h r p 2$ and $p f h r p 3$ as previously described [6]. All seven Loreto clinical isolates in the pfhrp2-deleted panel and parasites from the 3BD5 culture line were confirmed to be negative for both pfhrp 2 and $p f h r p 3$, while the D10 and Dd2 parasites were confirmed to be $p f h r p 2$-negative/pfhrp3-positive.

The three culture-adapted parasites lines were grown to between 1 and $2 \%$ parasitaemia using standard culture techniques [25], harvested and frozen at $-70{ }^{\circ} \mathrm{C}$. After determination of antigen (HRP2, Pf-LDH and aldolase) concentration by ELISA in the stock parasite preparations (methods below), frozen parasites were diluted using PCR-confirmed malaria negative group $\mathrm{O}$ blood. Eleven dilutions of each culture strain were generated with Pf-LDH concentration distributions similar to those in the wild-type panel (Table 1). A higher priority was 
Table 1 Characteristics of parasite panels used to test malaria rapid diagnostic tests

\begin{tabular}{|c|c|c|c|c|c|}
\hline \multirow[t]{2}{*}{ Panel } & \multirow[t]{2}{*}{ No. samples } & \multirow[t]{2}{*}{ Characteristics } & \multicolumn{2}{|c|}{ Antigen concentration $(\mathrm{ng} / \mathrm{mL})$} & \multirow[t]{2}{*}{ RDTs tested } \\
\hline & & & HRP2 & Pf-LDH & \\
\hline Wild-type & 100 & $\begin{array}{l}\text { Panel composition: } 100 \text { diluted clinical samples each } \\
\text { at } 200 \text { parasites/ } \mu \mathrm{L} \\
\text { pfhrp2 status: confirmed pfhrp2-positive } \\
\text { Sample origins: Central African Republic }(n=1) \text {, } \\
\text { Colombia }(n=6) \text {, Ethiopia }(n=1) \text {, Kenya }(n=1) \text {, } \\
\text { Cambodia }(n=17) \text {, Myanmar }(n=1) \text {, Nigeria } \\
(n=51) \text {, Peru }(n=6) \text {, Philippines }(n=1) \text {, Senegal } \\
(n=5) \text { and Tanzania }(n=10)\end{array}$ & $\begin{array}{l}\text { Mean: 11.76; } \\
\text { Median: 6.76; } \\
\text { Range: 0.67- } \\
62.48\end{array}$ & $\begin{array}{l}\text { Mean: 16.13; Median: } \\
\text { 13.59; Range: 0.19-53.53 }\end{array}$ & All \\
\hline pfhrp2-deleted & 40 & 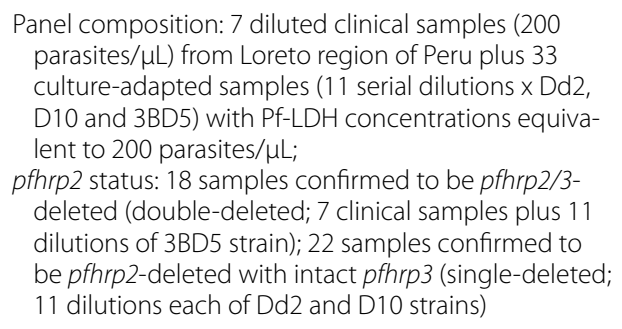 & $\begin{array}{l}\text { Mean: } 0.27 ; \\
\text { Median: } 0.11 ; \\
\text { Range: } \\
\text { 0.00-1.70; } \\
\text { Range (single- } \\
\text { deleted): } 0.10- \\
\text { 1.70; } \\
\text { Range (double- } \\
\text { deleted): } \\
\text { 0.00-0.20 }\end{array}$ & $\begin{array}{l}\text { Mean: 13.75; Median: 9.85; } \\
\text { Range: 2.50-58.00 }\end{array}$ & All \\
\hline $\begin{array}{l}\text { Double-deleted } \\
\text { (higher } \\
\text { density) }\end{array}$ & 18 & $\begin{array}{l}\text { Panel composition: } 7 \text { diluted clinical samples (2000 } \\
\text { parasites/ } \mu \mathrm{L} \text { ) from Loreto region of Peru plus } 11 \\
\text { samples of culture-adapted 3BD5 with tenfold } \\
\text { higher Pf-LDH concentrations than used in pfhrp2- } \\
\text { deleted panel } \\
\text { pfhrp2 status: confirmed pfhrp2/3-deleted }\end{array}$ & $\begin{array}{l}\text { Mean }^{\text {a. }}: 0.07 \\
\text { Median }^{\text {a }}: 0.00 \\
\text { Range }^{\text {a. }} \text { 0.00-0.37 }\end{array}$ & $\begin{array}{l}\text { Mean }^{\mathrm{a}}: 224.25 \\
\text { Median }^{\mathrm{a}}: 193.78 \\
\text { Range }^{\mathrm{a}}: \text { 47.50-526.00 }\end{array}$ & $\begin{array}{l}\text { RDTs with } \\
\text { Pf-LDH } \\
\text { test band }\end{array}$ \\
\hline
\end{tabular}

$R D T$ rapid diagnostic test, $H R P 2$ histidine-rich protein $2, P f$ - LDH P. falciparum lactate dehydrogenase

a Values calculated for clinical samples only. Antigen concentrations were not determined for higher density culture samples because the levels were measured for the same samples at a 1:10 dilution for the pfhrp2-deleted panel

given to matching the Pf-LDH distribution between panels because of the dominance of RDTs using this antigen, compared to aldolase (only one Round 8 product targeted aldolase).

A supplemental panel of double-deleted clinical and culture parasites at higher density was also produced using high density stocks of the same double-deleted parasite samples as in the pfhrp2-deleted panel, using the methods above (Table 1). This panel consisted of the seven clinical isolates from Peru, diluted to 2,000 parasites $/ \mu \mathrm{L}$ [26], and the 11 samples of the 3DB5 strain at tenfold higher concentration than used in the pfhrp2deleted panel described above.

\section{Measurement of antigen concentrations}

HRP2 and Pf-LDH concentrations in the stock and diluted samples of the pfhrp2-deleted panel were measured by commercial ELISA following manufacturers' instructions; Malaria Antigen Cellisa kit (Celllabs PTY LTD, Brookvale, NSW, Australia) for HRP2 and Qualisa malaria antigen pLDH ELISA kit (Tulip Diagnostics Ltd, Alto Santacruz, Goa, India) for Plasmodium-pLDH. Antigen concentrations of HRP2 and Pf-LDH were determined based on a standard curve run on each plate produced from serially diluted recombinant HRP2 and
Pf-LDH antigens, respectively. In addition, samples with known concentrations of antigen were run as internal controls for the assay.

The aldolase determinations were done using an inhouse ELISA. Capture (M/B 7-20) and detection antibodies (mAb C/D 11-4) were obtained from the National Bioproducts Institute (Pinetown, South Africa). The detection antibody mAb C/D 11-4 was biotinylated using EZ-Link Sulfo-NHS-Biotin (ThermoFisher Scientific, Waltham, MA, USA). Recombinant $P$. falciparum aldolase antigen (Microcoat Biotechnologie $\mathrm{GmbH}$, Germany), diluted in human malaria-negative blood was used to generate a standard curve from which aldolase concentrations were determined. For all ELISAs, each sample was run in duplicate, three or more times, on consecutive days and the antigen concentration determined based on the average of three runs.

\section{RDT testing procedure}

Each RDT product was tested against the wild-type and pfhrp2-deleted panels, with each sample tested in duplicate on two product lots by trained technicians blind to the randomized sample order. RDT band intensities were noted using a colour intensity chart as per the RDT Product Testing Standard Operating 
Procedures and results were double-entered into the WHO Product Testing database [26].

The RDT products targeting Pf-LDH were also tested against the double-deleted (higher-density) parasite panel. This testing was only conducted on one product lot, independent of testing the low-density wild-type and pfhrp2-deleted panels.

\section{RDT characteristics and categorization}

Thirty-two RDTs from 17 manufacturers were assessed. These were a sub-set of the 34 RDTs tested during Round 8 of the WHO Malaria RDT Product Testing Programme [4]; only RDTs with a false-positive rate below $10 \%$ against parasite-negative samples were included in the current study. The included RDTs are listed in four groups in Table 2 according to the target antigens

Table 2 Malaria RDT products included in evaluation

\begin{tabular}{|c|c|c|c|}
\hline Manufacturer & Product name & Product code & Target antigen(s) \\
\hline \multicolumn{4}{|l|}{ Pf-LDH detection RDTs (Group 1) } \\
\hline Access Bio, Inc. & CareStart ${ }^{\mathrm{TM}}$ Malaria Pf (HRP2/pLDH) Ag Combo 3-line RDT & RMSM-02571 & Pf-LDH, HRP2 \\
\hline Access Bio, Inc. & CareStart ${ }^{\mathrm{TM}}$ Malaria Pf (HRP2/pLDH) Ag RDT & RMPM-02571 & Pf-LDH/HRP2 \\
\hline Access Bio, Inc. & CareStart ${ }^{\mathrm{TM}}$ Malaria Pf/PAN (pLDH) Ag RDT & RMLM-02571 & Pan-LDH, Pf-LDH \\
\hline Access Bio Ethiopia & CareStart ${ }^{\mathrm{TM}}$ Malaria Pf (HRP2/pLDH) Ag RDT & RMPM-02591 & Pf-LDH/HRP2 \\
\hline WELLS BIO, INC & careUS ${ }^{\mathrm{TM}}$ Malaria Combo Pf (HRP2/pLDH) Ag & RMP-M02582 & Pf-LDH/HRP2 \\
\hline Advy Chemical Pvt. Ltd. & EzDx Malaria Pf Rapid malaria Antigen detection test (pLDH) & RK MAL 024-25 & Pf-LDH \\
\hline Meril Diagnostics Pvt Ltd. & MERISCREEN Malaria pLDH Ag & MVLRPD-02 & Pan-LDH, Pf-LDH \\
\hline Standard Diagnostics Inc. (Alere) & SD BIOLINE Malaria Ag P.f (HRP2/pLDH) & 05FK90 & Pf-LDH, HRP2 \\
\hline Standard Diagnostics Inc. (Alere) & SD BIOLINE Malaria Ag P.f/P.f/P.v & 05FK120 & Pf-LDH, Pv-LDH, HRP2 \\
\hline \multicolumn{4}{|l|}{ Pan-LDH only (Group 2) } \\
\hline Access Bio Ethiopia & CareStart ${ }^{\mathrm{TM}}$ Malaria PAN (pLDH) Ag RDT & RMNM-02591 & Pan-LDH \\
\hline WELLS BIO, INC & careUS $^{\mathrm{TM}}$ Malaria PAN (pLDH) Ag & RMN-M02582 & Pan-LDH \\
\hline \multicolumn{4}{|l|}{ HRP2-only (Group 3) } \\
\hline Access Bio, Inc. & CareStart ${ }^{\mathrm{TM}}$ Malaria Pf (HRP2) Ag RDT & RMOM-02571 & HRP2 \\
\hline Orchid Biomedical Systems (Tulip Group) & Paracheck Pf ${ }^{\circledR}$ Rapid Test for Pf Malaria (Ver. 3) & 302030025 & HRP2 \\
\hline SD Biosensor & STANDARD Q Malaria P.f Ag Test & 09MAL10B & HRP2 \\
\hline Omega Diagnostics Ltd. & VISITECT ${ }^{\circledR}$ Malaria Pf & OD336 & HRP2 \\
\hline \multicolumn{4}{|l|}{ HRP2-combination (Group 4) } \\
\hline ASPEN Laboratories PVT.LTD & Aspen ${ }^{\circledR} \mathrm{Mal}(\mathrm{Ag}$ Pf/Pv) Rapid Card Test & AS1550E & PV-LDH, HRP2 \\
\hline Access Bio, Inc. & CareStart ${ }^{\mathrm{TM}}$ Malaria Pf/PAN (HRP2/pLDH) Ag Combo RDT & RMRM-02571 & Pan-LDH, HRP2 \\
\hline Access Bio, Inc. & CareStart ${ }^{\mathrm{TM}}$ Malaria Pf/NOM (HRP2/pLDH) Ag Combo RDT & RMWM-02571 & Pvom-LDH, HRP2 \\
\hline Access Bio, Inc. & CareStart $^{\mathrm{TM}}$ Malaria Pf/Pv (HRP2/pLDH) Ag Combo RDT & RMVM-02571 & PV-LDH, HRP2 \\
\hline Access Bio Ethiopia & CareStart ${ }^{\mathrm{TM}}$ Malaria Pf/PAN (HRP2/pLDH) Ag Combo RDT & RMRM-02591 & Pan-LDH, HRP2 \\
\hline WELLS BIO, INC & careUS ${ }^{\mathrm{TM}}$ Malaria Combo Pf/PAN (HRP2/pLDH) Ag & RMR-M02582 & Pan-LDH, HRP2 \\
\hline Assure Tech (Hangzhou) & Ecotest Malaria P.f/Pan Rapid Test Device & MAL-W23M & Pan-LDH-HRP2 \\
\hline Nantong Egens Biotechnology Co., Ltd. & EGENS Malaria Pv/Pf Test Cassette & MAL-W23M (p.f/p.v) & PV-LDH, HRP2 \\
\hline Zephyr Biomedicals & FalciVax ${ }^{\mathrm{TM}}$ Rapid Test for Malaria Pv/Pf & 503010025 & Pv-LDH, HRP2 \\
\hline Premier Medical Corporation Private Ltd. & First Response ${ }^{\circledR}$ Malaria Ag. P.f./P.v. Card test ${ }^{c}$ & PI19FRC25 & PV-LDH, HRP2 \\
\hline Karwa Enterprises pvt Itd & Karwa ${ }^{\circledR} \mathrm{Mal}(\mathrm{Ag}$ Pf/Pv) Rapid Card Test & KW 1550E & PV-LDH, HRP2 \\
\hline Hangzhou AllTest Biotech Co. Ltd. & Malaria P.f./Pan Rapid Test Cassette & IMPN-402 & pan-aldolase, HRP2 \\
\hline Meril Diagnostics Pvt Ltd. & MERISCREEN Malaria Pf/Pan Ag & MHLRPD-02 & Pan-LDH, HRP2 \\
\hline Nectar Lifesciences Limited & Necviparum One Step Malaria P.f./P.v. Antigen Test & MAGDR & PV-LDH, HRP2 \\
\hline Zephyr Biomedicals & Parascreen ${ }^{\circledR}$ Rapid Test for Malaria Pan/Pf & 503030025 & Pan-LDH, HRP2 \\
\hline SD Biosensor & STANDARD Q Malaria P.f/Pan Ag Test & O9MAL30B & Pan-LDH, HRP2 \\
\hline SD Biosensor & STANDARD Q Malaria P.f/P.v Ag Test & 09MAL20B & PV-LDH, HRP2 \\
\hline
\end{tabular}

Target antigens captured by each test band are separated by a comma; target antigens captured on the same test band are indicated using a forward slash (/). HRP2: histidine-rich protein 2; Pf-LDH: P. falciparum lactate dehydrogenase; Pan-LDH: pan lactate dehydrogenase; Pv-LDH: P. vivax lactate dehydrogenase; Pvom-LDH: $P$, vivax, ovale and malariae lactate dehydrogenase 
detected and the a priori expected detection of $p f h r p 2-$ deleted parasites:

1. Group 1 RDTs detecting $P$. falciparum using Pf-LDH alone or in combination with other antigens $(n=9)$; expected to detect and correctly identify pfhrp2deleted P. falciparum.

2. Group 2 RDTs that detect $P$. falciparum using pan$\mathrm{LDH}$ alone $(\mathrm{n}=2)$; expected to detect pfhrp2-deleted P. falciparum as a Plasmodium positive sample.

3. Group 3 RDTs that detect $P$. falciparum only using HRP2 $(\mathrm{n}=4)$; expected to return false-negative results against $p$ fhrp2-deleted $P$. falciparum samples.

4. Group 4 Combination RDTs that detect $P$. falciparum using HRP2-only and other Plasmodium spp using pan or species-specific LDH, or aldolase $(n=17)$; expected to return false-negative results for falciparum infection against pfhrp2-deleted $P$. falciparum samples but false-positive results for non-falciparum infection (pan band positive, P. falciparum-specific band negative) when pan-LDH is used for one of the test lines.

Of the nine Group 1 (Pf-LDH) RDTs, three were dualband products with separate test bands detecting PfLDH and HRP2, and six were single-band products using either Pf-LDH alone, or a combination of Pf-LDH and HRP2 on the same band.

RDT positivity rate was defined as the percentage of valid tests that returned a positive result on the test band for P. falciparum (Pf band) or a positive result for Plasmodium in pan-only RDTs. The RDT positivity rate is equivalent to (100-false-negative rate). Valid tests were those which returned a positive control band. Since all samples were PCR-confirmed as P. falciparum only, any positive $P$. vivax or Pvom test line, or any positive pan test line in the absence of a positive Pf band, represents a false positive for non-falciparum infection. The nonfalciparum false positivity rate was the percentage of tests that returned a false-positive result for non-falciparum infection. It was not possible to determine non-falciparum false-positivity rates for pan-only (Group 2) and Pf-only (Group 3) RDTs because they do not differentiate species or have the capacity to detect non-falciparum infections, respectively.

\section{Statistical analysis}

This study reports descriptive statistics only. No formal statistical testing was conducted due to the small number of RDTs in each RDT group and the small number of samples within the pfhrp2-deleted panel, especially when separated into single and double-deleted samples.

\section{Results}

RDT positivity rates against pfhrp2-deleted panel compared to wild-type panel

The overall positivity of RDTs against pfhrp2-deleted parasites was $40.1 \%$, differing by RDT group: $57.1 \%$ for Group 1 (Pf-LDH), 95.6\% for Group 2 (pan-LDH only), 43.4\% for Group 3 (HRP2-only), and 23.7\% for Group 4 (HRP2-combination). The positivity rates were lower than against the wild-type panel, especially for Group 3 (HRP2-only) and Group 4 (HRP2-combination) RDTs (Table 3). There was wide variability in the positivity of Group 1 (Pf-LDH) RDTs, with product-specific positivity being similar between the double and single-deleted parasites, but lower than wild-type parasites (Table 3, Fig. 1). Large differences in positivity were observed between the double-deleted, single-deleted and wild-type parasites for Group 3 (HRP2-only) and 4 (HRP2-combination) RDTs, and Group 4 RDTs also showed large inter-product variation in Pf band positivity against single-deleted P. falciparum parasites (Table 3, Fig. 1). The positivity of individual

Table 3 RDT Pf band positivity against pfhrp2-deleted and wild type panels according to RDT group

\begin{tabular}{|c|c|c|c|}
\hline \multirow[t]{3}{*}{ RDT Group } & \multicolumn{3}{|c|}{ Median positivity ${ }^{a}(\%)(\min -\max )$} \\
\hline & \multicolumn{2}{|c|}{ Pfhrp2-deleted panel } & \multirow{2}{*}{$\begin{array}{l}\text { Wild type panel } \\
\left(n=400^{b}\right)\end{array}$} \\
\hline & $\begin{array}{l}\text { Double-deleted } \\
\left(\mathrm{n}=72^{\mathrm{b}}\right)\end{array}$ & $\begin{array}{l}\text { Single-deleted } \\
\left(\mathrm{n}=\mathbf{8 8}^{\mathrm{b}}\right)\end{array}$ & \\
\hline $1(\mathrm{Pf}-\mathrm{LDH})(\mathrm{n}=9)$ & $63.9(13.9-86.1)$ & $59.1(11.4-84.1)$ & $93.8(30.5-99.0)$ \\
\hline 2 (pan-LDH only) $(n=2)$ & $95.1(94.4-95.8)$ & $96.0(94.3-97.7)$ & $99.5(99.5-99.5)$ \\
\hline 3 (HRP2-only) $(n=4)$ & $6.3(0.0-15.3)$ & $69.9(61.4-92.0)$ & $96.0(91.5-98.0)$ \\
\hline 4 (HRP2-combination) $(n=17)$ & $0.0(0.0-20.8)$ & $35.2(1.1-80.7)$ & $92.5(70.3-98.2)$ \\
\hline
\end{tabular}

For Group 2 (pan-LDH only) RDTs the pan band is used to determine positivity

a Positivity was calculated for each RDT against the relevant parasite panel; median, minimum and maximum positivity were then calculated across the RDTs within each group, respectively. Only valid RDT results are included in positivity calculations

b Each sample in each panel was tested against four RDTs of the same product 


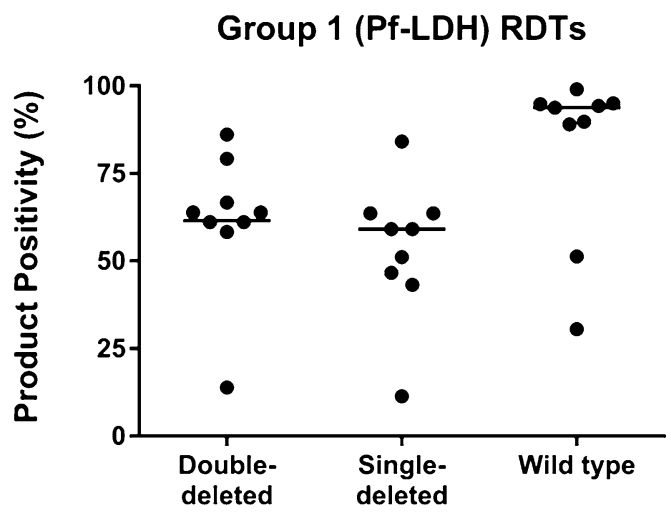

Sample Type

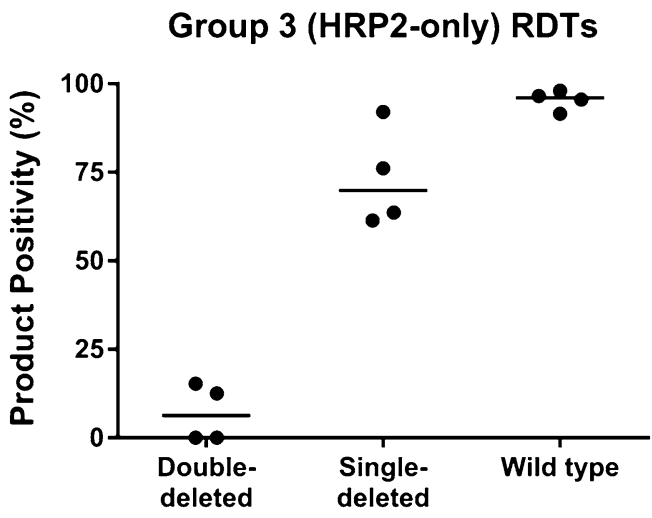

Sample Type

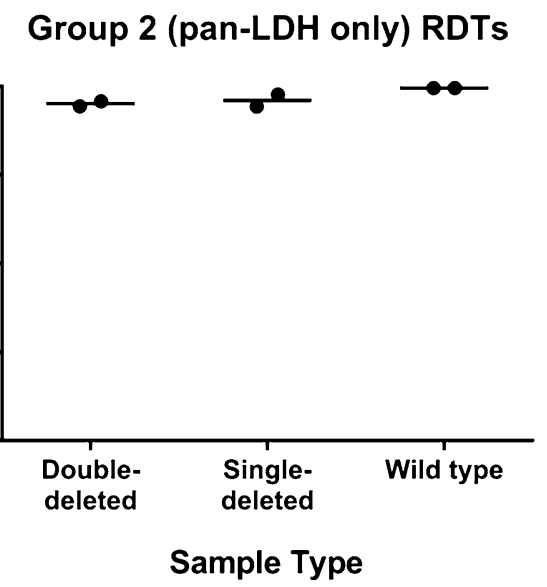

Group 4 (HRP2-combination) RDTs

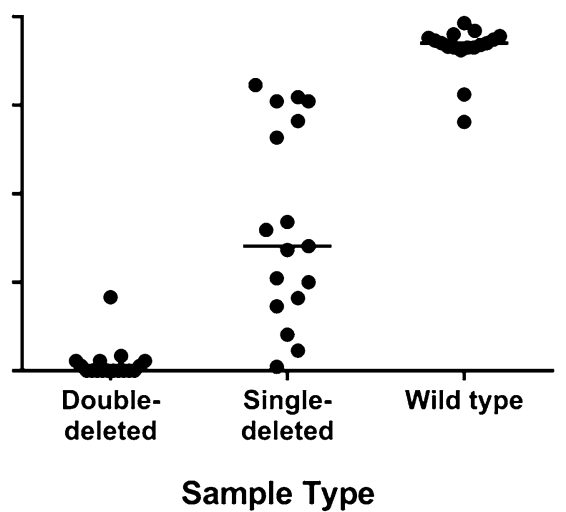

Fig. 1 RDT positivity for individual products according to RDT group and sample type Lines indicate the median positivity for each RDT group against each sample type

products and lots are available from the Round $8 \mathrm{WHO}$ malaria RDT product testing report [4].

Three Group 1 (Pf-LDH) RDTs had a separate HRP2detecting test band, in addition to the Pf-LDH test band. The positivity of this HRP2 band ranged from 0.0 to $1.4 \%$ against double-deleted parasites and 0.0 to $12.5 \%$ against single-deleted P. falciparum.

\section{Plasmodium falciparum test band intensity}

Where positive results on the Pf bands of RDTs were obtained against the pfhrp2-deleted panel, the band intensities were weak (Figs. 2 and 3). Considering the HRP2-detecting bands only, Group 3 (HRP2-only) and Group 4 (HRP2-combination) RDTs had a higher positivity (band intensity $>0$ ) against single deleted parasites than did the Group 1 (Pf-LDH) RDTs (Fig. 2).

There was some evidence of differences in band intensities for the Pf-LDH bands of the Group 1 (Pf-LDH) RDTs between products that used Pf-LDH alone and those that also contained an independent HRP2 test line (Fig. 3), however the number of RDTs was too small for statistical testing. The most noticeable difference was for the six RDTs that detected P. falciparum using Pf-LDH alone, or on a combined Pf-LDH/HRP2 test line, where there was a lower band intensity against the double- and single-deleted parasites in the pfhrp2-deleted panel compared to the wild-type panel (Fig. 3, right panel). In contrast, none of the three RDTs that contained independent HRP2 and Pf-LDH test bands achieved an intensity on the Pf-LDH test band above 2 on any parasite panel (Fig. 3, left panel).

\section{False positives for non-falciparum infection}

The non-falciparum false positivity rates for Group 4 (HRP2-combination) RDTs were elevated for some products against the pfhrp2-deleted panel, compared to the wild-type panel, as evidenced by the large difference in maximum false-positivity rates between the different 

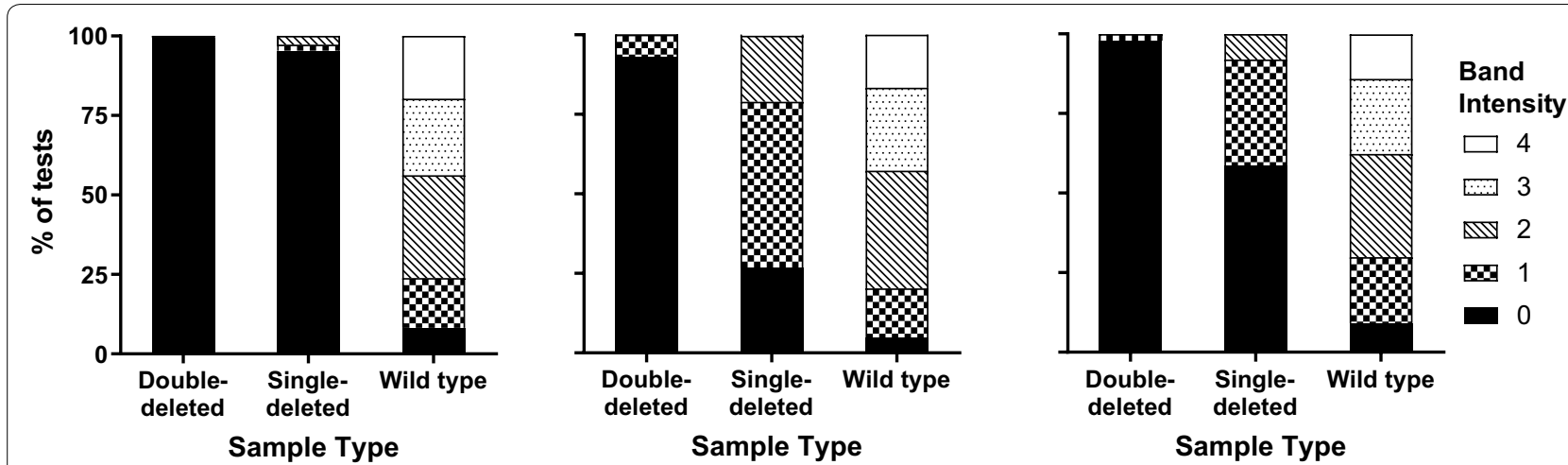

Fig. 2 Distribution of HRP2 test band intensities according to sample type for Group 1 (Pf-LDH) RDTs which contained an independent HRP2 band $(n=3)$ (left), Group 3 (HRP2-only) RDTs $(n=4)$ (center) and Group 4 (HRP2-combination) RDTs ( $=17)$ (right)

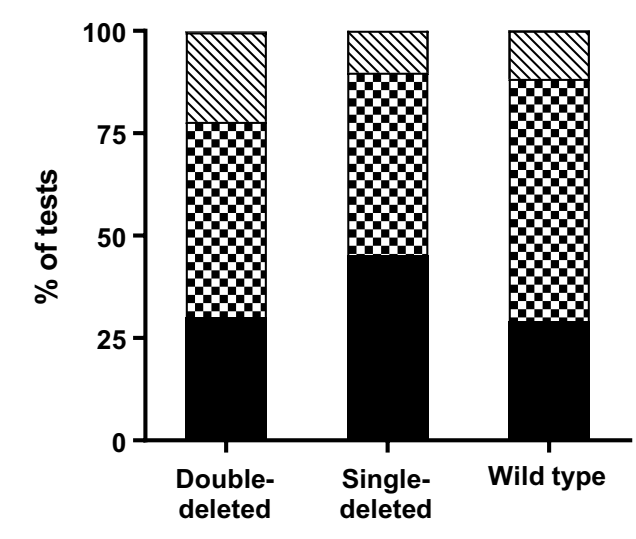

Sample Type

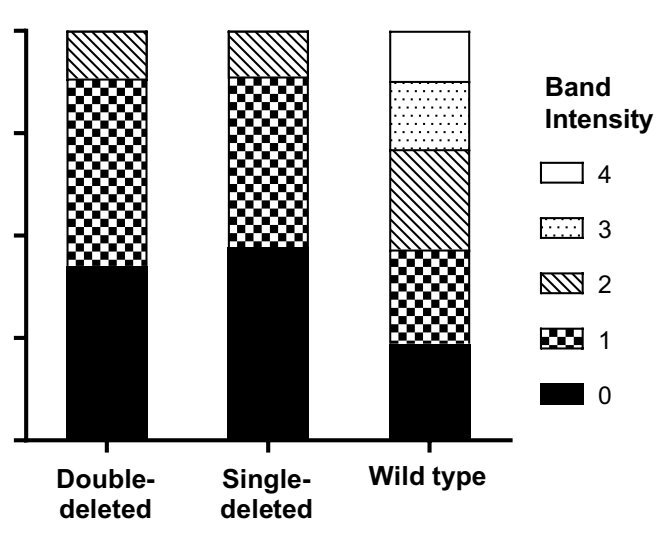

Sample Type

Fig. 3 Distribution of Pf-LDH test band intensity for Group 1 (Pf-LDH) RDTs according to sample type: RDTs using both HRP2 and Pf-LDH as independent test bands to detect P. falciparum $(\mathrm{n}=3$ ) (left); RDTs only using Pf-LDH or a combined HRP2/Pf-LDH test band to detect $P$. falciparum $(n=6)$ (right)

sample types (Table 4). A similar pattern was observed with two of the three combination Group 1 (Pf-LDH) RDTs, with one product returning a non-falciparum false-positive rate of $55.6 \%$ against the double-deleted $P$. falciparum parasites (Table 4). Details of non-falciparum false positives for individual products can be found in the Round 8 WHO malaria RDT product testing report [4].

\section{Performance of Pf-LDH RDTs against double-deleted clinical and culture samples at higher antigen \\ concentrations}

The performance of the Group 1 (Pf-LDH) RDTs was assessed against the double-deleted (higher density) panel. Eight RDTs had a positivity of $100 \%$ against the seven clinical double-deleted samples, while one RDT (Product D) returned positive results on six of the seven (85.7\%) samples. The mean band intensity against these clinical samples was 2.8 with some products showing
Table 4 Non-falciparum false positivity rates for combination RDTs in Groups 1 (Pf-LDH) and 4 (HRP2combination)

\begin{tabular}{llll}
\hline RDT Group $^{\mathbf{a}}$ & \multicolumn{2}{l}{$\begin{array}{l}\text { Median non-falciparum false positivity rate } \\
\text { (min-max) }\end{array}$} \\
\cline { 2 - 4 } & $\begin{array}{l}\text { Double- } \\
\text { deleted } \\
\text { samples } \\
(\mathbf{n}=\mathbf{7 2})\end{array}$ & $\begin{array}{l}\text { Single- } \\
\text { deleted } \\
\text { samples } \\
(\mathbf{n}=\mathbf{8 8})\end{array}$ & $\begin{array}{l}\text { Wild type samples } \\
(\mathbf{n}=\mathbf{4 0 0})\end{array}$ \\
\hline $1(\mathrm{Pf}-\mathrm{LDH})(\mathrm{n}=3)$ & $13.9(0-55.6)$ & $21.6(0-34.1)$ & $2.0(0-10.3)$ \\
$4(\mathrm{HRP2-Combina-}$ & $1.4(0-81.9)$ & $1.1(0-43.2)$ & $0.8(0-3.0)$ \\
tion) $(\mathrm{n}=17)$ & & & \\
\hline
\end{tabular}

a RDT Groups 2 (pan-LDH only) and 3 (HRP2-only) are not included as it was not possible to determine non-falciparum false positivity rates because these RDTs do not differentiate species or have the capacity to detect non-falciparum infections, respectively

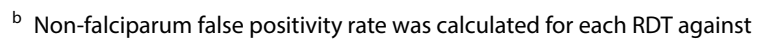
the relevant parasite panel; median, minimum and maximum values were then calculated across the RDTs within each group. Only valid RDT results are included in non-falciparum false positivity rate calculations 
a high proportion of strong (3 or 4) band intensities (Fig. 4). One of the three combination Group 1 RDTs returned one non-falciparum false-positive result (14.3\%) on the pan-LDH band. The band intensity of positive tests was higher against the higher density doubledeleted clinical isolates compared to the same samples at lower density (Table 5). Similar results were obtained against the higher density culture panel; eight RDTs had a positivity of $100 \%$, while one (Product D) only detected $45 \%$ of the samples (Fig. 4 ).

\section{Discussion}

The recent emergence of $p$ fhrp2/3-deleted parasites in several African and South American countries, as well as India, has rapidly escalated the need for RDTs that are not solely reliant on HRP2 for the detection of $P$. falciparum. Modelling studies have shown that use of RDTs reliant only on HRP2 detection can exert selective pressure on the parasite population to drive the spread of pfhrp2/3-deleted P. falciparum [27, 28]. The WHO recommends that countries do not exclusively rely on HRP2-based RDTs where the prevalence of $p f h r p 2$ deletions causing false-negative RDTs is greater than $5 \%$ in symptomatic patients [29]. In many cases this would be operationalized by changing from a HRP2-detecting RDT to a pan-LDH and/or Pf-LDH-detecting RDT, with the assumption that these RDTs perform equally well on HPR2-negative and HRP2-positive parasites. However, this assumption had not been previously tested and the current results suggest that performance of Pf-LDHdetecting RDTs against wild-type samples do not predict performance against pfhrp2/3-deleted parasites (clinical and cultured samples).
Table 5 Pf-LDH band intensities for positive RDTs in Group 1 (Pf-LDH) against double-deleted clinical samples

\begin{tabular}{|c|c|c|c|c|}
\hline \multirow[t]{2}{*}{ Product } & \multicolumn{2}{|c|}{200 parasites $/ \mu \mathrm{l}(\mathrm{n}=28)$} & \multicolumn{2}{|c|}{2000 parasites $/ \mu \mathrm{l}(n=14)$} \\
\hline & Mean & Median (min-max) & Mean & Median (min-max) \\
\hline$A$ & 1.45 & $1.0(1-3)$ & 3.57 & $4.0(1-4)$ \\
\hline B & 1.52 & $2.0(1-2)$ & 3.21 & $3.0(2-4)$ \\
\hline C & 1.50 & $1.5(1-2)$ & 2.71 & $3.0(1-4)$ \\
\hline $\mathrm{D}$ & 1.00 & $1.0(1-1)$ & 2.08 & $2.0(2-3)$ \\
\hline$E$ & 1.33 & $1.0(1-2)$ & 2.86 & $3.0(2-4)$ \\
\hline $\mathrm{F}$ & 1.17 & $1.0(1-2)$ & 2.43 & $2.5(1-4)$ \\
\hline G & 1.17 & $1.0(1-2)$ & 2.50 & $2.5(1-4)$ \\
\hline $\mathrm{H}$ & 1.52 & $2.0(1-2)$ & 2.86 & $3.0(2-3)$ \\
\hline I & 1.55 & $2.0(1-2)$ & 3.29 & $3.0(2-4)$ \\
\hline
\end{tabular}

Positive RDTs are those with a Pf-LDH band intensity of at least 1 on the colour intensity chart included in the RDT Product Testing Standard Operating Procedures

There was large variability in the positivity of the nine Pf-LDH RDTs tested against samples equivalent to 200 parasites $/ \mu \mathrm{L}$, but as a group they unexpectedly appeared to detect wild-type $P$. falciparum at higher rates than pfhrp2-deleted parasites. Indeed, one combination PfLDH RDT assessed in this study met the WHO performance criteria against wild-type $P$. falciparum with a Panel Detection Score (PDS) of 83 (89\% positivity), but obtained a PDS of 0 (12\% positivity) when assessed against pfhrp2-deleted parasites [4]. Antigen concentration is a potential confounder in the comparison between performance against wild-type and pfhrp2-deleted parasites, so the pfhrp2-deleted panel was prepared to have a similar distribution of Pf-LDH concentration to the wild-type panel, with all ELISAs run in triplicate using

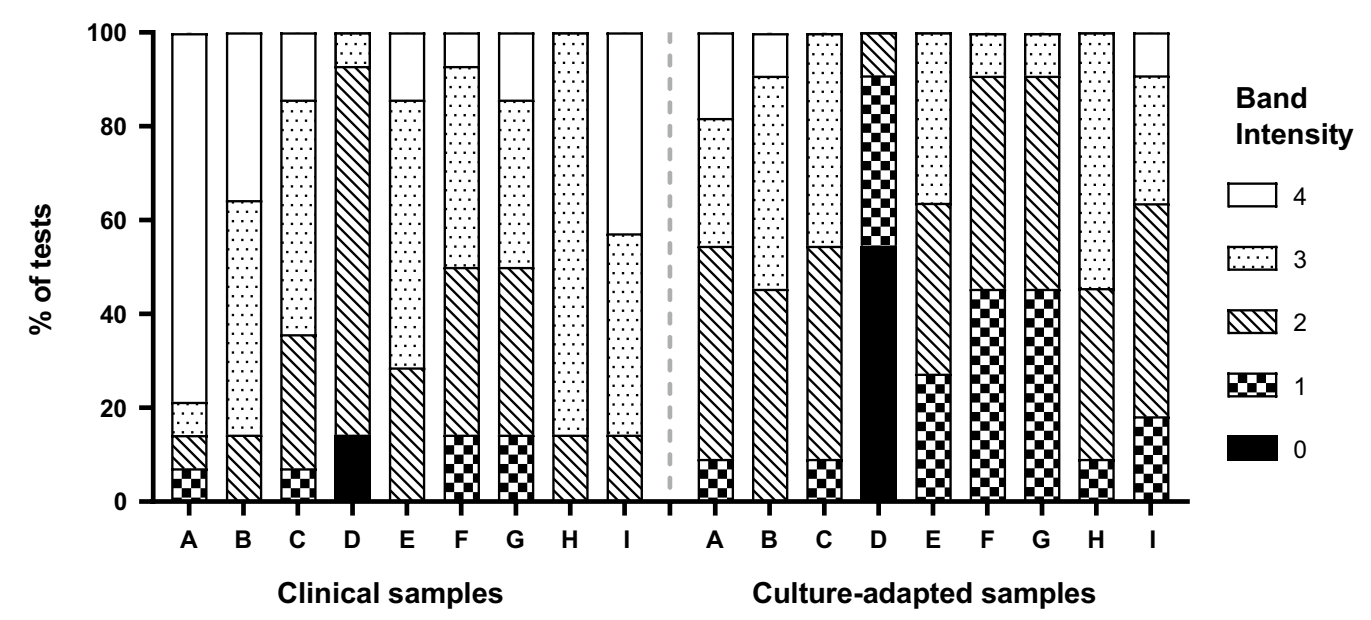

Fig. 4 Distribution of Pf-LDH band intensities of the nine Group 1 (Pf-LDH) RDTs, labelled A to I, when tested against the double-deleted (higher density) panel containing seven clinical samples (left) and 11 culture-adapted samples (right) 
the same controls for each panel. Indeed, if the decreased positivity were due to variation in Pf-LDH concentration, it would be expected that the pan-LDH RDTs would show comparable decreases in performance when challenged against the pfhrp2-deleted parasites, which was not the case. Therefore, it is unlikely that differences in antigen concentrations explain the observed results.

The products using Pf-LDH included both dual-band products, with separate test bands detecting Pf-LDH and HRP2, and single-band products, using either PfLDH alone or a combination of Pf-LDH and HRP2 on the same band. Interestingly, the reduced performance on the $p f h r p 2$-deleted panel compared to wild-type panel appeared to be restricted to Pf-LDH detecting products that did not contain a separate HRP2 band. This may be a spurious result due to the small number of PfLDH-detecting RDTs examined, or the limited size and diversity of the $p$ fhrp2-deleted panel or product specific issues, such as Pf-LDH test lines unexpectedly reacting with HRP2. Reassuringly, all Pf-LDH RDTs were able to detect a small set of double-deleted clinical isolates at the higher parasite density of 2,000 parasites $/ \mu \mathrm{L}$.

Although assessments against larger, more geographically diverse panels are needed, these results suggest that where good quality microscopy is not available and where the prevalence of pfhrp2/3-deleted parasites leading to false-negative RDT results is $>5 \%$ [29], the pan-LDH RDTs would be suitable for P. falciparum detection. The two pan-LDH-only products had the best performance against pfhrp2-deleted parasites and well exceeded the minimum WHO RDT performance criteria for P. falciparum, specifically $>75 \%$ panel detection score at 200 parasites $/ \mu \mathrm{L}$. However, neither of these products is yet WHO pre-qualified, nor are they in the assessment pipeline, and this may limit procurement by certain agencies [30]. The one pan-LDH-only RDT that is currently WHO prequalified has not been evaluated against a pfhrp2-deleted panel and these current results highlight the need for additional assessments.

On the other hand, in areas that require differentiation of $P$. falciparum from non-P. falciparum infection for treatment decision making, reporting or surveillance, current Pf-LDH-detecting products could have utility until better RDTs become available. The risk-benefit of presumptive treatment of fever versus false-negative PfLDH RDTs secondary to parasitaemia below 2,000 parasites $/ \mu \mathrm{L}$ would need to be carefully considered.

Although the main focus of this study was to assess the performance of Pf-LDH-detecting RDTs, a variety of RDTs that only detect $P$. falciparum using HRP2 were also included. This provided the opportunity to review how these products respond with the a priori assumption that HRP2-detecting RDTs would not detect pfhrp2-deleted parasites, an assumption which was confirmed for parasites lacking both $p f h r p 2$ and $p f h r p 3$. The majority of field studies test for the presence of both $p f h r p 2$ and $p f h r p 3$ but to date very few have found $p f h r p 2$-negative/pfhrp3positive parasites [22]. However, in this study both double- and single-deleted parasites were included, since the structural similarity between HRP2 and HRP3 may provide the opportunity for cross-reactivity [3]. The results demonstrate that some, but not all, HRP2-detecting RDTs return positive results against single-deleted $P$. falciparum at concentrations equivalent to 200 parasites/ $\mu \mathrm{L}$. Hence, it appears that some products tested are able to detect HRP3, as previously reported, and even at lower concentrations $[6,22]$. Therefore, the risk of incorrect diagnosis posed by single-deleted mutants is reduced when certain RDT brands are used. In this study, detection of pfhrp2-negative/pfhrp3-positive parasites for individual HRP2-based RDTs ranged from very limited (1.1\%) to almost complete detection $(92.0 \%)$. This large variation has implications for the detection and surveillance of HRP2-negative parasites, since in some cases only double-deleted parasites will present as RDTnegative, while in other cases both single- and doubledeleted parasites will present as RDT-negative. This difference could affect the frequency of false-negative RDT results and also survey estimates of the prevalence of pfhrp2-deleted parasites in symptomatic patients, if HRP2-negative RDT results are used as a first screen for mutant parasites that require genotyping [31]. Therefore, buyers should consider these results when selecting an HRP2 RDT to purchase, as the cross-reactivity afforded by HRP3 will reduce the number of false-negative RDT results. For surveillance, estimation of the prevalence of pfhrp2-deleted parasites may require inclusion of a subset of HRP2-positive RDTs from malaria patients, as well as genotyping for both $p f h r p 2$ and $p f h r p 3$. Furthermore, it is not known if $p f h r p 2$ single-deleted mutants are a harbinger for pfhrp3 deletions, and subsequently the double deletions that generate false-negative results on HRP2 test lines.

Combination RDTs that use HRP2 to detect P. falciparum and pan-LDH to detect Plasmodium spp are widely used in areas where $P$. falciparum and $P$. vivax co-exist. These tests potentially detect HRP2-negative parasites but are likely to misclassify the result as a non-falciparum infection. The results of this study demonstrate that there is large variability in the rate of this type of false positivity between products, a feature that is possibly related to the specific antibody used and dependent on whether the HRP2 band cross-reacts with HRP3, as well as the sensitivity of the pan-LDH test band. The variability in the positivity of the pan-LDH band noted in this study matches that previously reported [32], and suggests 
reliance on the pan-LDH band to detect HRP2-negative parasites in regions where $P$. falciparum dominates may be unreliable.

An important limitation of this study is the small panel size, particularly when separated into double- and singledeleted samples. Unfortunately, all the single-deleted samples were prepared from two culture lines, as no clinical samples were available. There appears to be no difference in RDT performance against clinical and culture samples in the double-deleted parasites used, suggesting that the use of culture-derived samples has not significantly impacted the results of the study.

\section{Conclusion}

The current study demonstrates that Pf-LDH detecting RDTs respond strongly to high-density $P$. falciparum samples lacking $p f h r p 2$, but performance at lower densities is variable. It is recommended that further testing of Pf-LDH detecting RDTs be conducted against a larger and geographically diverse panel of HRP2-negative samples. Surprisingly, many HRP2-detecting RDTs were able to detect single-deleted parasites at low density, a likely positive outcome for clinical management of $P$. falciparum in areas where pfhrp2-negative/pfhrp3-positive parasites exist, but a potential source of discrepancy for reporting prevalence of HRP2-negative parasites. Ultimately, new targets for $P$. falciparum detection should be explored, especially since optimizing Pf-LDH RDTs has proven difficult for manufacturers, so clinicians and community health workers can have confidence in RDT results to make clear treatment decisions in all malaria endemic regions.

\section{Abbreviations}

CDC: US Centers for Disease Control and Prevention; HRP2: Histidine-rich protein 2; HRP3: Histidine-rich protein 3; Pf: Plasmodium falciparum; Pv: Plasmodium vivax; Pvom: Plasmodium vivax, P. ovale, P. malariae; LDH: Lactate dehydrogenase; RDTs: Rapid diagnostic tests; WHO: World Health Organization.

\section{Acknowledgements}

The authors thank Tom Wellems for supply of the culture-adapted parasite 3BD5 under a material transfer agreement between the US National Institutes of Health $(\mathrm{NIH})$ and the Centers for Disease Control and Prevention (CDC), and Dionicia Gamboa and Katherine Torres from Universidad Peruana Cayetano Heredia, Lima, Peru for supply of clinical pfhrp2-deleted isolates. Yong Ah, Scott Wilson and Jeffrey Glenn were supported by a grant from FIND to the CDC Foundation.

\section{Authors' contributions}

MLG contributed to the study design, data analysis and interpretation and was a major contributor in writing the manuscript. AC cleaned the data and performed the data analysis. JG, SW and YA prepared the parasite material, conducted the laboratory testing and data collection and cleaning. AK was involved in preparation of parasite material. RLO and RRR performed antigen quantification. PC and SI were involved in design of the study. QC was involved with the design of the study, literature searching and was a major contributor to writing the manuscript. MA was involved with the design of the study, overseeing the laboratory testing and drafting the manuscript. JC was involved with designing the study and was a major contributor in writing the manuscript. All authors read and approved the final manuscript.

\section{Funding}

This work was supported by the Foundation for Innovative New Diagnostics via a grant from UNITAID. The funder had no role in the design or conduct of this work, preparation of the manuscript or the decision to publish.

\section{Availability of data and materials}

The data generated or analysed during this study are either included in this published article or available from the World Health Organization publication Malaria rapid diagnostic test performance: results of WHO product testing of malaria RDTs: round 8 (2016-2018) [https://www.who.int/malaria/publications/ atoz/9789241514965/en/].

\section{Ethics approval and consent to participate}

Each collection site for the clinical isolates obtained approval from a WHO research ethics review committee and/or a local institutional review board for specimen collection, transport and archiving of blood samples for the purpose of RDT product testing, lot testing and quality assurance.

\section{Consent for publication \\ Not applicable.}

\section{Competing interests}

MLG, RRR, AC, JG, SW and YA report grants from Foundation for Innovative New Diagnostics during the conduct of the study; MLG also reports personal fees from World Health Organization; QC, SI, RLO, PC, MA, JC and AK have nothing to disclose.

\begin{abstract}
Author details
${ }^{1}$ Queensland University of Technology, Brisbane, QLD, Australia. ${ }^{2}$ The CDC Foundation, Atlanta, GA, USA. ${ }^{3}$ Centers for Disease Control and Prevention, Atlanta, USA. ${ }^{4}$ Hospital for Tropical Diseases, London, UK. ${ }^{5}$ London School of Hygiene and Tropical Medicine, London, UK. ${ }^{6}$ Foundation for Innovative New Diagnostics (FIND), Geneva, Switzerland. ${ }^{7}$ Australian Defence Force Malaria and Infectious Diseases Institute (FORMERLY Australian Army Malaria Institute), Brisbane, QLD, Australia. ${ }^{8}$ World Health Organization, Geneva, Switzerland.
\end{abstract}

Received: 27 May 2020 Accepted: 23 October 2020

Published online: 04 November 2020

\section{References}

1. WHO. Guidelines for the treatment of malaria. 3rd Edn. Geneva, World Health Organization, 2015.

2. WHO. World malaria report 2019. Geneva, World Health Organization, 2019.

3. Rock EP, Marsh K, Saul AJ, Wellems TE, Taylor DW, Maloy WL, et al. Comparative analysis of the Plasmodium falciparum histidine-rich proteins HRP-I, HRP-II and HRP-III in malaria parasites of diverse origin. Parasitology. 1987;95:209-27.

4. WHO. Malaria rapid diagnostic test performance: results of WHO product testing of malaria RDTs: round 8 (2016-2018). Geneva, World Health Organization, 2018. https://www.who.int/malaria/publications/ atoz/9789241514965/en/. Accessed 7 August 2019.

5. Akinyi S, Hayden T, Gamboa D, Torres K, Bendezu J, Abdallah JF, et al. Multiple genetic origins of histidine-rich protein 2 gene deletion in Plasmodium falciparum parasites from Peru. Sci Rep. 2013;3:2797.

6. Gamboa D, Ho MF, Bendezu J, Torres K, Chiodini PL, Barnwell JW, et al. A large proportion of $P$. falciparum isolates in the Amazon region of Peru lack pfhrp2 and pfhrp3: implications for malaria rapid diagnostic tests. PLoS One. 2010;5:e8091.

7. Maltha J, Gamboa D, Bendezu J, Sanchez L, Cnops L, Gillet P, et al. Rapid diagnostic tests for malaria diagnosis in the Peruvian Amazon: impact of pfhrp2 gene deletions and cross-reactions. PLoS ONE. 2012;7:e43094.

8. Murillo Solano C, Akinyi Okoth S, Abdallah JF, Pava Z, Dorado E, Incardona $\mathrm{S}$, et al. Deletion of Plasmodium falciparum Histidine-Rich Protein 2 
(pfhrp2) and Histidine-Rich Protein 3 (pfhrp3) genes in Colombian Parasites. PLOS ONE. 2015;10:e0131576.

9. Akinyi Okoth S, Abdallah JF, Ceron N, Adhin MR, Chandrabose J, Krishnalall $\mathrm{K}$, et al. Variation in Plasmodium falciparum histidine-rich protein 2 (Pfhrp2) and Plasmodium falciparum histidine-rich protein 3 (Pfhrp3) gene deletions in Guyana and Suriname. PLoS ONE. 2015;10:e0126805.

10. Rachid Viana GM, Akinyi Okoth S, Silva-Flannery L, Lima Barbosa DR, Macedo de Oliveira A, Goldman IF, et al. Histidine-rich protein 2 (pfhrp2) and pfhrp3 gene deletions in Plasmodium falciparum isolates from select sites in Brazil and Bolivia. PLoS One. 2017;12:e0171150.

11. Houze S, Hubert V, Le Pessec G, Le Bras J, Clain J. Combined deletions of pfhrp2 and pfhrp3 genes result in Plasmodium falciparum malaria falsenegative rapid diagnostic test. J Clin Microbiol. 2011;49:2694-6.

12. Fontecha G, Mejia RE, Banegas E, Ade MP, Mendoza L, Ortiz B, et al. Deletions of pfhrp2 and pfhrp3 genes of Plasmodium falciparum from Honduras Guatemala and Nicaragua. Malar J. 2018;17:320.

13. Li P, Xing H, Zhao Z, Yang Z, Cao Y, Li W, et al. Genetic diversity of Plasmodium falciparum histidine-rich protein 2 in the China-Myanmar border area. Acta Trop. 2015;152:26-31.

14. Kumar N, Pande V, Bhatt RM, Shah NK, Mishra N, Srivastava B, et al. Genetic deletion of HRP2 and HRP3 in Indian Plasmodium falciparum population and false negative malaria rapid diagnostic test. Acta Trop. 2013;125:119-21.

15. Bharti PK, Chandel HS, Ahmad A, Krishna S, Udhayakumar V, Singh N. Prevalence of pfhrp2 and/or pfhrp3 gene deletion in Plasmodium falciparum Population in eight highly endemic states in India. PLOS ONE. 2016;1 1:e0157949.

16. Koita OA, Doumbo OK, Ouattara A, Tall LK, Konare A, Diakite M, et al. False-negative rapid diagnostic tests for malaria and deletion of the histidine-rich repeat region of the hrp2 gene. Am J Trop Med Hyg. 2012:86:194-8.

17. Amoah LE, Abankwa J, Oppong A. Plasmodium falciparum histidine rich protein-2 diversity and the implications for PfHRP 2: based malaria rapid diagnostic tests in Ghana. Malar J. 2016;15:101.

18. Wurtz N, Fall B, Bui K, Pascual A, Fall M, Camara C, et al. Pfhrp2 and pfhrp3 polymorphisms in Plasmodium falciparum isolates from Dakar, Senegal: impact on rapid malaria diagnostic tests. Malar J. 2013;12:34.

19. Parr JB, Verity R, Doctor SM, Janko M, Carey-Ewend K, Turman BJ, et al. Pfhrp2-deleted Plasmodium falciparum parasites in the Democratic Republic of Congo: a national cross-sectional survey J Infect Dis. 2017;216:36-44.

20. Kozycki CT, Umulisa N, Rulisa S, Mwikarago El, Musabyimana JP, Habimana JP, et al. False-negative malaria rapid diagnostic tests in Rwanda: impact of Plasmodium falciparum isolates lacking hrp2 and declining malaria transmission. Malar J. 2017;16:123.

21. Laban NM, Kobayashi T, Hamapumbu H, Sullivan D, Mharakurwa S, Thuma PE, et al. Comparison of a PfHRP2-based rapid diagnostic test and PCR for malaria in a low prevalence setting in rural southern Zambia: implications for elimination. Malar J. 2015;14:25.

22. Beshir KB, Sepulveda N, Bharmal J, Robinson A, Mwanguzi J, Busula $\mathrm{AO}$, et al. Plasmodium falciparum parasites with histidine-rich protein 2 (pfhrp2) and pfhrp3 gene deletions in two endemic regions of Kenya. Sci Rep. 2017;7:14718.

23. Berhane A, Anderson K, Mihreteab S, Gresty K, Rogier E, Mohamed S, et al. Major Threat to malaria control programs by Plasmodium falciparum lacking Histidine-Rich Protein 2 Eritrea. Emerg Infect Dis. 2018;24:462-70.

24. WHO. Response Plan for pfhrp $2 / 3$ deletions. Geneva, World Health Organization, 2019. https://www.who.int/malaria/publications/atoz/ response-plan-pfhrp2-gene-deletions/en/. Accessed 7 August 2019.

25. Trager W, Jensen JB. Human malaria parasites in continuous culture. Science. 1976;193:673-5.

26. Foundation for Innovative New Diagnostics (FIND), WHO Global Malaria Programme, United States Centers for Disease Control and Prevention (CDC). Methods manual for product testing of malaria rapid diagnostic tests. Version 7 2018. http://www.who.int/malaria/publications/rdtmanual/en/. Accessed 7 Aug 2019.

27. Gatton ML, Dunn J, Chaudhry A, Ciketic S, Cunningham S, Cheng $\mathrm{Q}$ Implications of parasites lacking Plasmodium falciparum Histidine-Rich Protein 2 on malaria morbidity and control when rapid diagnostic tests are used for diagnosis. J Infect Dis. 2017;215:1156-66.

28. Watson OJ, Slater HC, Verity R, Parr JB, Mwandagalirwa MK, Tshefu A et al. Modelling the drivers of the spread of Plasmodium falciparum hrp2 gene deletions in sub-Saharan Africa. eLife. 2017;6:e25008.

29. WHO Global Malaria Programme: False-negative RDT results and implications of new reports of $P$. falciparum histidine-rich protein $2 / 3$ gene deletions. Geneva, World Health Organization, 2017. https://www.who, int/malaria/publications/atoz/information-note-hrp2-based-rdt/en/. Accessed 7 August 2019.

30. WHO. Malaria: progress of the active applications in the prequalification of IVDs assessment pipeline. Geneva, World Health Organization, 2018. http://www.who.int/diagnostics_laboratory/180808_malaria.pdf?ua=1. Accessed 21 November 2018.

31. WHO. Surviellance template protocol for pfhrp $2 / 3$ gene deletions. Geneva, World Health Organization, 2020. https://www.who.int/malaria/ publications/atoz/hrp2-deletion-protocol/en/. Accessed 2 October 2020

32. Gatton ML, Rees-Channer RR, Glenn J, Barnwell JW, Cheng Q, Chiodini PL, et al. Pan-Plasmodium band sensitivity for Plasmodium falciparum detection in combination malaria rapid diagnostic tests and implications for clinical management. Malar J. 2015;14:115

\section{Publisher's Note}

Springer Nature remains neutral with regard to jurisdictional claims in published maps and institutional affiliations.
Ready to submit your research? Choose BMC and benefit from:

- fast, convenient online submission

- thorough peer review by experienced researchers in your field

- rapid publication on acceptance

- support for research data, including large and complex data types

- gold Open Access which fosters wider collaboration and increased citations

- maximum visibility for your research: over $100 \mathrm{M}$ website views per year

At BMC, research is always in progress.

Learn more biomedcentral.com/submissions 